\title{
TRIM22 activates NF-KB signaling in glioblastoma by accelerating the degradation of ІкBa
}

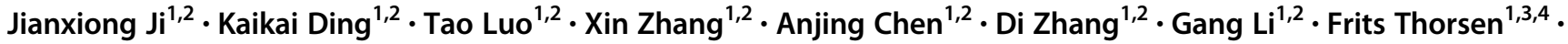 \\ Bin Huang ${ }^{1,2} \cdot$ Xingang $\mathrm{Li}^{1,2} \cdot$ Jian Wang $\mathbb{B}^{1,2,3}$
}

Received: 25 December 2019 / Revised: 29 July 2020 / Accepted: 6 August 2020 / Published online: 19 August 2020

(c) The Author(s) 2020. This article is published with open access

\begin{abstract}
NF- $\mathrm{KB}$ signaling plays a critical role in tumor growth and treatment resistance in GBM as in many other cancers. However, the molecular mechanisms underlying high, constitutive NF- $\mathrm{kB}$ activity in GBM remains to be elucidated. Here, we screened a panel of tripartite motif (TRIM) family proteins and identified TRIM22 as a potential activator of NF- $\mathrm{kB}$ using an NF- $\mathrm{kB}$ driven luciferase reporter construct in GBM cell lines. Knockout of TRIM22 using Cas9-sgRNAs led to reduced GBM cell proliferation, while TRIM22 overexpression enhanced proliferation of cell populations, in vitro and in an orthotopic xenograft model. However, two TRIM22 mutants, one with a critical RING-finger domain deletion and the other with amino acid changes at two active sites of RING E3 ligase (C15/18A), were both unable to promote GBM cell proliferation over controls, thus implicating E3 ligase activity in the growth-promoting properties of TRIM22. Co-immunoprecipitations demonstrated that TRIM22 bound a negative regulator of NF- $\mathrm{KB}, \mathrm{NF}-\mathrm{\kappa B}$ inhibitor alpha ( $\mathrm{I} \kappa \mathrm{B} \alpha)$, and accelerated its degradation by inducing K48-linked ubiquitination. TRIM22 also formed a complex with the NF- $\mathrm{KB}$ upstream regulator IKK $\gamma$ and promoted K63linked ubiquitination, which led to the phosphorylation of both IKK $\alpha / \beta$ and I $\mathrm{KB} \alpha$. Expression of a non-phosphorylation mutant, srIאB $\alpha$, inhibited the growth-promoting properties of TRIM22 in GBM cell lines. Finally, TRIM22 was increased in a cohort of primary GBM samples on a tissue microarray, and high expression of TRIM22 correlated with other clinical parameters associated with progressive gliomas, such as wild-type IDH1 status. In summary, our study revealed that TRIM22

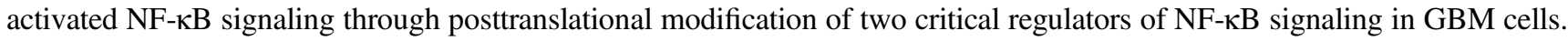

These authors contributed equally: Jianxiong Ji, Kaikai Ding

Edited by V. D'Angiolella

Supplementary information The online version of this article (https:// doi.org/10.1038/s41418-020-00606-w) contains supplementary material, which is available to authorized users.

Bin Huang

hb@sdu.edu.cn

$\triangle$ Xingang Li

lixg@sdu.edu.cn

$\triangle$ Jian Wang

jian.wang@uib.no

1 Department of Neurosurgery, Qilu Hospital and Institute of Brain and Brain-Inspired Science, Cheeloo College of Medicine, Shandong University, Jinan, China

2 Shandong Key Laboratory of Brain Function Remodeling, Jinan, China

3 Department of Biomedicine, University of Bergen, Jonas Lies vei 91, 5009 Bergen, Norway

4 Molecular Imaging Center, University of Bergen, Jonas Lies vei 91, 5009 Bergen, Norway

\section{Introduction}

The NF- $\mathrm{kB}$ pathway, originally characterized within the context of the immune system, has been implicated in many hallmarks of cancer development, including cellular proliferation, angiogenesis, and resistance to therapy [1-3]. As in other malignancies, high, constitutive NF- $\mathrm{KB}$ activity has been observed in human glioblastoma (GBM). It has been found to promote mesenchymal differentiation and therapy resistance in the disease [4], and also plays a central role in many other active oncogenic pathways in GBM [5-10]. Expression profiling and molecular analysis has demonstrated that GBMs with mesenchymal features exhibit elevated levels of NF- $\mathrm{kB}$ pathway genes, such as RELB and TRADD [11]. However, mutation or amplification of NF- $\mathrm{kB}$ signaling subunits is rare in tumors, suggesting that aberrant activation of NF- $\mathrm{kB}$ signaling in GBM may be attributed to deregulation of the pathway or oncogenes.

Ubiquitination, one of the most common and important types of posttranslational modification, has been implicated 
in many diseases including cancers $[12,13]$. The most wellstudied polyubiquitin chain types include lysine 48 (K48) and lysine 63 (K63) linkages. K48-linked polyubiquitin chains predominantly target proteins for degradation, while K63 chains regulate kinase activity, signal transduction, and endocytosis $[14,15]$. Over the past decade, posttranslational modification, especially ubiquitin (Ub) modification, has emerged as a crucial participant in NF- $\mathrm{kB}$ activation [16]. Following stimulation, signaling intermediaries, such as TNF receptor-associated factors and receptor interacting protein, are rapidly modified with K63-linked poly-Ub chains, leading to activation of downstream kinases, such as TGF- $\beta$-activated kinase 1 and IKK complexes that participate in NF-кB signaling. The IKK complex is made up of two catalytic subunits (IKK $\alpha$ and IKK $\beta$ ) and a noncatalytic regulatory subunit (IKK $\gamma$ ) [16-20]. Transcriptional activation of NF- $\mathrm{KB}$ occurs when the IKK complex becomes activated and phosphorylates IKB proteins. Phosphorylation of I $\mathrm{B} \alpha$ frees NF- $\kappa \mathrm{B}$, which is translocated to the nucleus, while phosphorylated I $\mathrm{I} B \alpha$ is targeted for degradation after modification with K48-linked Ub chains [21].

One subfamily of the RING type E3 Ub ligases, the tripartite motif (TRIM) proteins, is emerging as a key regulator in the development of diverse cancers by modulating transcriptional activity of NF- $\mathrm{kB}$, including GBM [13]. TRIM40 was reported to physically binds to NEDD8 and promotes the neddylation of IKK $\gamma$ to inhibit NF-KB activation in gastrointestinal carcinomas [22]. TRIM24 has also been shown to significantly alter transcriptional activity of NF-kB in EGFRvIII-driven GBM cells [23]. Here, using $\mathrm{NF}-\mathrm{\kappa B}$ response reporters and public databases, we further investigated the role of TRIM proteins in the development of GBM. We screened a panel of TRIM proteins using an $\mathrm{NF}-\mathrm{\kappa B}$ driven luciferase reporter construct and identified TRIM22 as an activator of NF-KB signaling in GBM cells. TRIM22, rarely investigated in most human cancers, regulates biological processes in various cell types through NF$\kappa \mathrm{B}$ signaling, including macrophages [24, 25], neurons [26], and human embryonic kidney $293 \mathrm{~T}$ cells (HEK293T) $[27,28]$. We found it not only promoted K48-linked ubiquitination of $\mathrm{I} \kappa \mathrm{B} \alpha$ by directly associating with it, but also enhanced K63-linked ubiquitination of IKK $\gamma$. Thus, TRIM22 both activates the IKK complex and promotes degradation of $\mathrm{I} \kappa \mathrm{B} \alpha$, thereby leading to high transcriptional activity of NF-kB in GBM cells.

\section{Materials and methods}

\section{Ethics statement}

All primary glioma tissue samples $(n=112)$ and matching clinical data were obtained from the Department of
Neurosurgery at Qilu Hospital of Shandong University [29]. Nonneoplastic brain tissue samples (NBT; $n=10$ ) were obtained from the Department of Pathology at Qilu Hospital of Shandong University. All experiments and the use of human tissues were approved by the Research Ethics Committee of Shandong University and the Ethics Committee of Qilu Hospital in accordance with the Declaration of Helsinki (for humans) and the U.S. Public Health Service Policy on Human Care and Use of Laboratory Animals (2015 reprint; for mice). Written informed consent was obtained from all adult patients.

\section{Cell culture}

Human GBM cell lines (U87MG, U118MG and LN229) and the human embryonic kidney cell line 293 (HEK293) were purchased from the American Type Culture Collection. All human cell lines were authenticated and submitted for short tandem repeat analysis (Cell Cook Biotech Co. LTD; Guangzhou, China). Primary GBM\#P3 cells and BG5 glioma stem cells (GSC) were kind gifts provided by Prof. Rolf Bjerkvig (University of Bergen, Norway). Cells (U87MG, U118MG, LN229, and HEK293) were maintained in the Dulbecco's modified Eagle's medium (DMEM; Life Technologies/Thermo Fisher Scientific; Waltham, MA, USA) supplemented with $10 \%$ fetal bovine serum (Thermo Fisher Scientific). Cells (GBM\#P3 and GSC BG5) were cultured in serum-free DMEM/F12 medium (Gibco/Thermo Fisher Scientific) supplemented with 2\% B27 Neuro Mix (Thermo Fisher Scientific), epidermal growth factor $(20 \mathrm{ng} / \mathrm{mL}$; Thermo Fisher Scientific), and basic fibroblast growth factor $(10 \mathrm{ng} / \mathrm{mL}$; Thermo Fisher Scientific). Cells were maintained at $37^{\circ} \mathrm{C}$ in a humidified chamber containing $5 \% \mathrm{CO}_{2}$.

\section{Transient transfection, lentiviral infection, and Cas9- sgRNA knockout}

Transient transfections for siRNAs and plasmids were performed with Lipofectamine 2000 or 3000 (Thermo Fisher Scientific) as we previously described [30]. The sequences of siRNAs used are listed in Supplementary Table S1, and plasmids used are listed in Supplementary Table S2.

The Puro-3 $\times$ Flag-hCas9 and single-guide RNA (sgRNAs) lentiviruses were designed and constructed by OBiO Technology Company (Shanghai, China). Lentivirus containing Cas9 and sgRNAs (sg-scramble, sg-TRIM22-1 or sg-TRIM22-2) were introduced into U87MG, LN229, GBM\#P3, and BG5 cells. The sequences of sgRNAs used are listed in Supplementary Table S1.

In addition, lentiviral constructs for ectopic expression of full-length TRIM22 (Flag-TRIM22-FL; OBiO Technology), a RING-finger domain deletion mutant of TRIM22 (FlagTRIM22- $\Delta$ RING; OBiO Technology), a C15/18A mutant of TRIM22 (Flag-TRIM22-C15/18A; OBiO Technology), 
and an $\mathrm{S} 32 / \mathrm{S} 36 \mathrm{~A}$ mutant of $I \kappa B \alpha(\operatorname{srI} \kappa \mathrm{B} \alpha ; \mathrm{OBiO}$ Technology) were also used to infect cells.

After $48 \mathrm{~h}$, infected cells were cultured in media containing puromycin $(2 \mu \mathrm{g} / \mathrm{mL}$; Thermo Fisher Scientific) for 2 weeks to select for stable expression.

\section{Subcellular fractionation}

Nuclear and cytoplasmic fractions from LN229 and U118MG were isolated using Nuclear and Cytoplasmic Extraction Reagents (Thermo Fisher Scientific), according to the manufacturer's instructions. Subcellular distribution of proteins was determined using western blot analysis. GAPDH and Histone H3 served as loading controls for cytosolic and nuclear fractions, respectively.

\section{Real-time quantitative RT-PCR (qRT-PCR)}

Total RNA was extracted from cells using TRIzol Reagent (Thermo Fisher Scientific). RNA $(2 \mu \mathrm{g})$ was reverse transcribed into cDNA using the ReverTra Ace qPCR RT Kit (Toyobo Life Science; Shanghai, China) according to the manufacturer's protocols. Quantitative PCR was performed using the SYBR premix Ex Taq (Takara; Tokyo, Japan) on the Real-Time PCR Detection System (480II, Roche; Basel, Switzerland). GAPDH served as the internal control. Primers used for PCR are listed in Supplementary Table S3.

\section{Immunohistochemistry (IHC), immunofluorescence (IF), and western blotting (WB)}

IHC, IF, and WB were performed as previously described [29]. The scoring system for TRIM22 IHC staining and all antibodies used are described in Supplementary Materials and Methods.

\section{Co-immunoprecipitation (Co-IP)}

Co-IPs were performed as previously described [29]. Briefly, cells were lysed in IP lysis buffer (Thermo Fisher Scientific) containing a protease inhibitor cocktail (1\%, Sigma-Aldrich; St Louis, MO, USA). Total lysates $(200 \mu \mathrm{g}$; $1 \mu \mathrm{g} / \mu \mathrm{L})$ were incubated with primary antibodies $(4 \mu \mathrm{L})$ or IgG $(4 \mu \mathrm{L})$ overnight at $4{ }^{\circ} \mathrm{C}$ with gentle shaking followed by Protein A/G magnetic beads (Thermo Fisher Scientific) for $2 \mathrm{~h}$ at room temperature. The immunoprecipitated complexes were immunoblotted. The antibodies used are listed in Supplementary Materials and Methods.

\section{Cycloheximide (CHX) chase}

LN229 and U87MG cells were infected with lentivirus containing Cas9 and sgRNAs targeting TRIM22 (OBiO
Technology). LN229 and U118MG cells were infected with lentivirus for ectopic expression of Flag-TRIM22-FL, FlagTRIM22-C15/18A, or Flag-TRIM22- $\Delta$ RING (OBiO Technology). After selection, CHX $(25 \mu \mathrm{g} / \mathrm{mL}$; Apexbio; Houston, TX, USA) was introduced to the culture medium to inhibit translation, and cell lysates were prepared at the indicated times. Protein $(20 \mu \mathrm{g})$ was examined using western blot analysis.

\section{Cell number counting}

Cells with target gene knockout or ectopic overexpression $\left(1 \times 10^{5} /\right.$ well $)$ were seeded into six-well plates. Cells were collected through trypsinization and counted every $24 \mathrm{~h}$. Cells were counted in three wells to obtain an average count, and each experiment was performed in three independent biological replicates.

\section{Luciferase reporter assays}

The NF- $\kappa$ B firefly-luciferase and renilla reporter constructs (100 ng each, Promega; Madison, WI, USA) were cotransfected into modified U87MG, U118MG, and LN229 cells using Lipofectamine 3000 (Thermo Fisher Scientific). After $24 \mathrm{~h}$, luciferase activities were examined using the Dual-Luciferase Reporter Assay Kit (Promega). Renilla activity was used to normalize luciferase reporter activity. The promoterless firefly-luciferase vector pGL4.15 served as the negative control (NC). Assays were performed on cells in three wells for each experiment to obtain an average count, and in three independent biological replicates.

\section{In vivo and in vitro ubiquitination assay}

To assess in vivo ubiquitination, modified cells were treated with $20 \mu \mathrm{M}$ MG132 (Apexbio; Houston, TX, USA) for $6 \mathrm{~h}$ before lysis, followed by co-IP and western blot analysis. For in vitro ubiquitination assays, $\mathrm{HA}-\mathrm{I} \kappa \mathrm{B} \alpha$ proteins purified from HEK293 cells were incubated with UBE1 (100 ng), UbcH5a (150 ng), human recombinant Ub (5 $\mu$, Boston Biochem; Cambridge, MA, USA) in the absence or presence of Flag-TRIM22-FL or Flag-TRIM22- $\Delta$ RING purified proteins with ubiquitination reaction buffer (Boston Biochem) at $30^{\circ} \mathrm{C}$ for $90 \mathrm{~min}$. Co-IPs were performed on the incubation mixture using an anti-HA antibody followed by western blot analysis using an anti-K48-linkage specific polyubiquitin antibody.

\section{Animal studies}

Athymic nude mice (male, 4-week old; GemPharmatech Co., Ltd; Nanjing, China) were randomly divided into five 
animals per group. Luciferase-expressing human glioma cell lines $\left(3 \times 10^{5}\right.$ cells suspended in $10 \mu \mathrm{L}$ PBS $)$ were implanted into the frontal lobes of nude mice using a stereotactic apparatus (KDS310, KD Scientific; Holliston, MA, USA). Tumor growth was examined at 6, 12, and 24 days after implantation using bioluminescence imaging (IVIS spectrum in vivo imaging system, PerkinElmer; Hopkinton, MA, USA). Animals were euthanized by cervical dislocation when they displayed any symptoms of continuous discomfort, such as severe hunchback posture, decreased activity, apathy, dragging legs, or more than $20 \%$ weight loss. Mouse brains were harvested and examined through hematoxylin and eosin and IHC/IF staining.

\section{Database (oncomine)}

Oncomine (https://www.oncomine.org/) was used to compare mRNA expression levels between GBM and nonneoplastic tissue samples for TRIM5, TRIM21, TRIM22, and TRIM38 in TCGA Brain datasets and TRIM56 in the Sun Brain dataset.

\section{Statistical analysis}

All experiments were performed in at least three independent biological replicates and reported as the mean \pm the standard error of the mean. The statistical significance was calculated utilizing an unpaired two-tailed Student's $t$ test for direct comparisons and ANOVA for multigroup comparisons. Survival curves were estimated using the Kaplan-Meier method and compared using the log-rank test. Correlation between TRIM22 expression levels and clinicopathological factors was determined using the twotailed $\chi^{2}$ test or the Fisher's exact test. Statistical analysis was conducted using GraphPad Prism version 7.00 software for Windows (GraphPad; La Jolla, CA, USA). Differences were considered as statistically significant when $P$ values were $<0.05$.

\section{Results}

\section{Identification of TRIM22 as a positive regulator of NF-KB signaling}

To identify novel NF- $\mathrm{KB}$ signaling modulators, we screened the activity of a panel of TRIM proteins using an NF- $\mathrm{KB}$ dependent transcriptional reporter containing five copies of an NF- $\mathrm{KB}$ response element (NRE) located upstream of luciferase. We chose to analyze five TRIM proteins, TRIM5, TRIM21, TRIM22, TRIM38, and TRIM56, in our luciferase assay based on two criteria. First, the expression levels of the genes, based on molecular data in public datasets in Oncomine, were significantly elevated in GBM relative to NBT samples (Supplementary Fig. S1A). Second, these five TRIM genes have not yet been reported to have a role in the development of human gliomas. We transfected siRNAs targeting each of these genes with the luciferase reporter construct into U87MG and LN229 cells and measured luciferase activity. TRIM22 siRNA decreased reporter activity the most among the five TRIM genes in both U87MG and LN229 cells compared with the NC group (Fig. 1a). Using CRISPR/Cas9 technology, we knocked out TRIM22 in both cell lines and found luciferase activity to be significantly reduced (Supplementary Fig. S2A).

To elucidate the mechanism underlying reduced NF- $\mathrm{KB}$ activity in TRIM22 knockout cells, we used western blot analysis to examine total protein and phosphorylated levels of core kinases involved in canonical NF- $\mathrm{kB}$ signaling. Phosphorylated levels of the proteins that examined IKK $\alpha / \beta$ (Ser176/180), IкB $\alpha$ (Ser32/36), and P65 (Ser536) were uniformly decreased in TRIM22 knockout cell lines. However, total protein levels remained unchanged, except in the case of IкB $\alpha$, where they increased in response to TRIM22 knockout (Fig. 1b and Supplementary Fig. S3A).

To understand how loss of TRIM22 might contribute to increased levels of I $\mathrm{K} \mathrm{B} \alpha$ protein, we first examined $I \kappa B \alpha$ mRNA levels. $I \kappa B \alpha$ mRNA levels did not change significantly with TRIM22 knockout in U87MG and LN229 cells (Fig. 1c). This result indicated that $\mathrm{I} \kappa \mathrm{B} \alpha$ protein might be stabilized in TRIM22 knockout cells. Therefore, we determined the half-life of $\mathrm{I} \kappa \mathrm{B} \alpha$ by treating modified U87MG and LN229 cells with CHX. The half-life of I $\mathrm{KB} \alpha$ was prolonged by $\sim 8 \mathrm{~h}$ in U87MG-sg-TRIM22 and LN229sg-TRIM22 cells compared to the sg-scramble groups (Fig. 1d, e). Finally, K48-linked ubiquitination of IкB $\alpha$ in control and modified U87MG and LN229 cells paralleled TRIM22 expression; endogenous K48-linked ubiquitination of IкB $\alpha$ was decreased with knockout of TRIM22 (Fig. 1f). Taken together, these data suggested that deletion of TRIM22 enhanced IкB $\alpha$ protein stability through loss of proteasomal-mediated protein degradation.

\section{TRIM22 deletion inhibits GBM cell proliferation}

NF- $\mathrm{KB}$ signaling is considered to be a pivotal factor in inducing genes that promote cell survival and proliferation [5]. Our previous work also demonstrated a growth-promoting role for $\mathrm{NF}-\mathrm{kB}$ in GBMs [29]. To test whether TRIM22 is involved in regulating GBM cell proliferation, growth curves were generated for TRIM22 knockout U87MG and LN229 cells and compared to controls in vitro. Growth curves generated over $72 \mathrm{~h}$ revealed that TRIM22 knockout significantly attenuated proliferation of U87MG and LN229 cells relative to control cell lines (Fig. 2a). Reduced Ki-67 IF staining in knockdown 
A

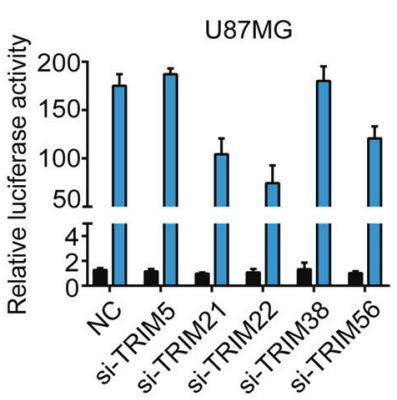

Negative control $\square$ NF-kB

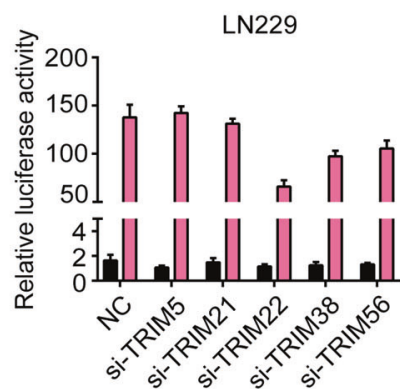

Negative control $\square$ NF-kB
B

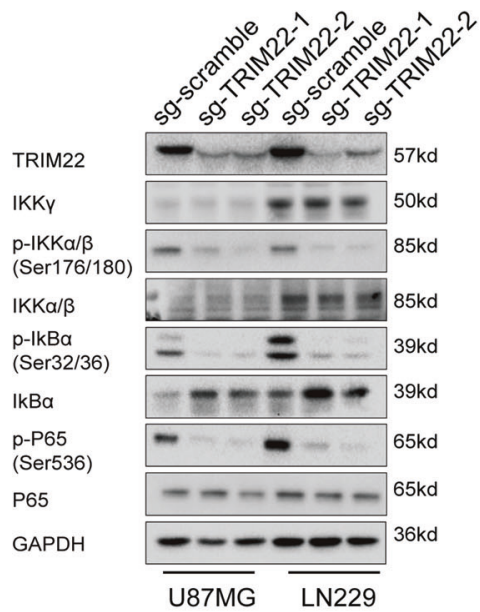

C

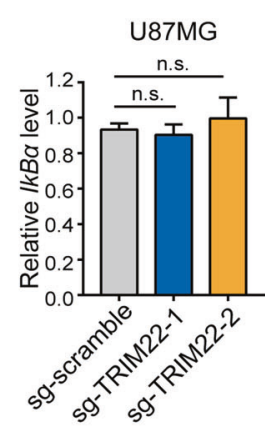

E

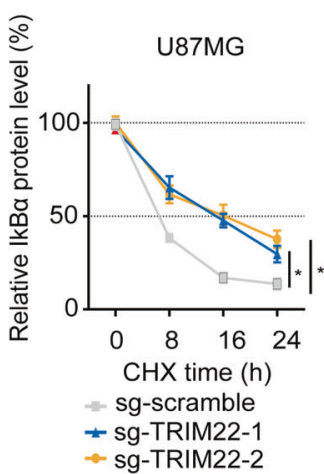

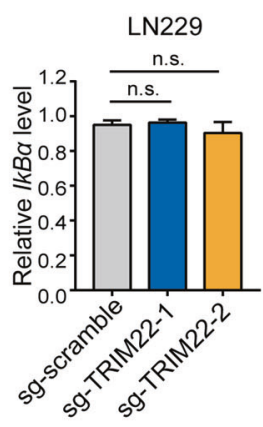

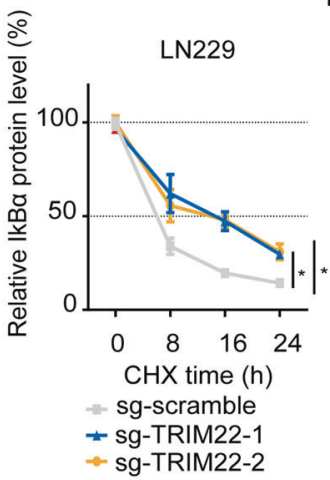

D
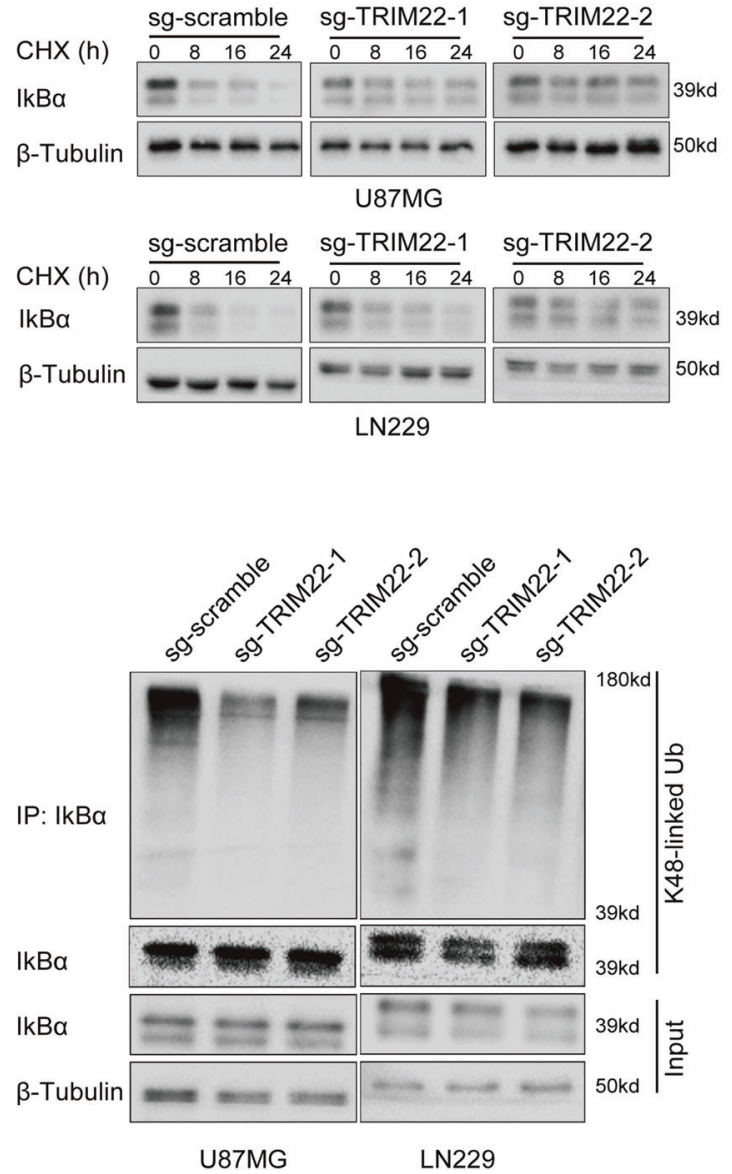

normalize samples. d Western blot analysis of $\mathrm{I} \kappa \mathrm{B} \alpha$ protein in TRIM22-depleted cells treated with cycloheximide (CHX; $25 \mu \mathrm{g} / \mathrm{mL}$ ) for $0,8,16$, and $24 \mathrm{~h}$. e Decay curve of I $\mathrm{I} B \alpha$ levels normalized to $\beta$-tubulin and to $0 \mathrm{~h}$ at the indicated time points from CHX experiments. f Western blot analysis of IPs performed with antibody to I $\mathrm{B} \alpha$ to detect endogenous I $\mathrm{I} B \alpha$ ubiquitination from indicated cells. Antibody for K48-linked polyubiquitin was used on the western blot. Student's $t$ test: n.s. not significant, $* P<0.05$.

We also examined tumor growth in vivo. Luciferaseexpressing modified and control cell lines were proliferation in vitro $(\sim 50 \%$; Fig. $2 b)$. 


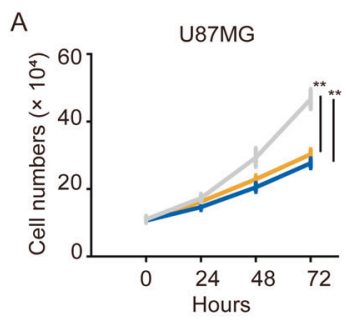

- sg-scramble

- sg-TRIM22-1

- sg-TRIM22-2

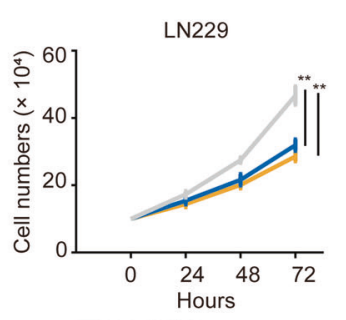

- sg-scramble

- sg-TRIM22-1

- sg-TRIM22-2
B

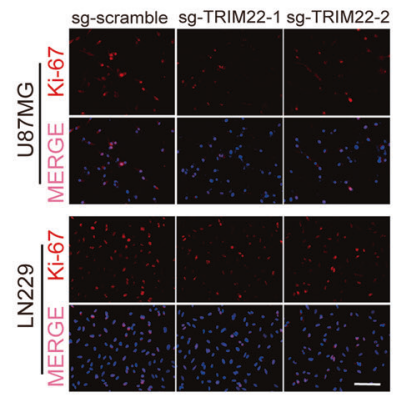

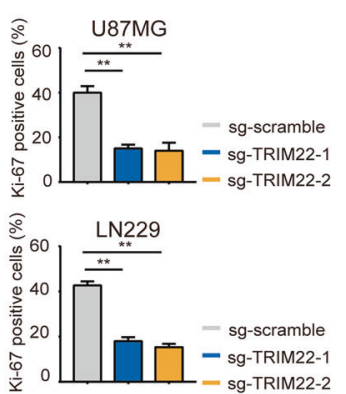

C

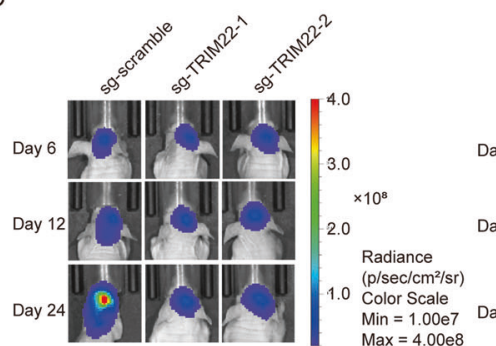

U87MG

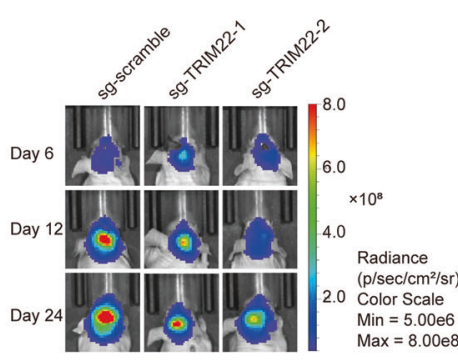

LN229
D

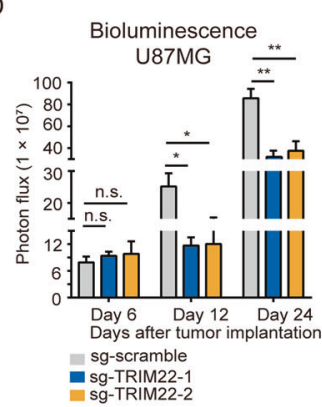

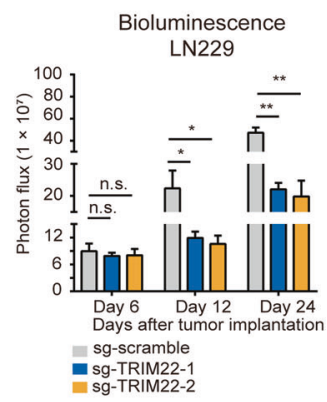

E
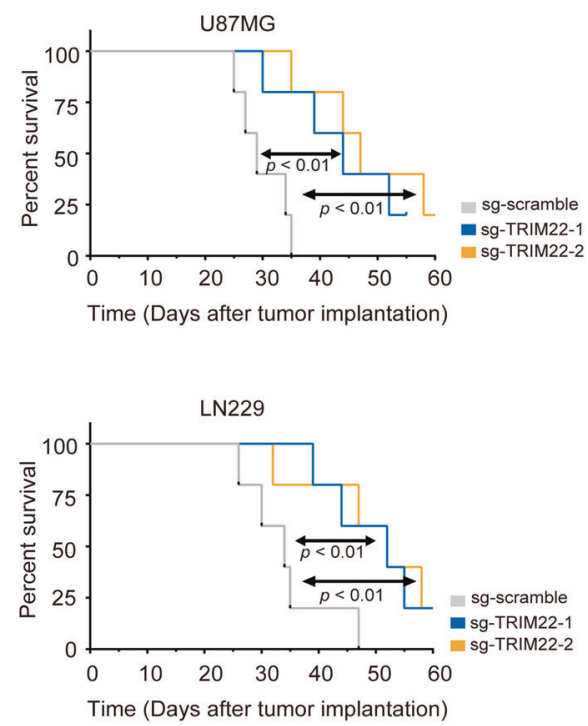

Fig. 2 Knockout of TRIM22 inhibits proliferation of GBM in vitro and in vivo. a Growth curves generated using cell counting over $72 \mathrm{~h}$ for the cells indicated. b Representative images and quantification of Ki-67 immunofluorescence staining from modified U87MG and LN229 cells. c, d Images and quantification of in vivo bioluminescence imaging of U87MG- and LN229-NC and -sg-TRIM22-1 and -2 derived xenografts at the indicated time points. e Kaplan-Meier

orthotopically implanted in mice, and tumor growth was monitored/quantified using bioluminescence. Tumors derived from U87MG- and LN229-sg-TRIM22-1 or -2 cells were slower growing than the sg-scramble control counterparts (Fig. 2c, d). Survival time of U87MG- and LN229-sg-TRIM22-1 or -2 tumor-bearing animals was also prolonged relative to controls (U87MG: 44 or

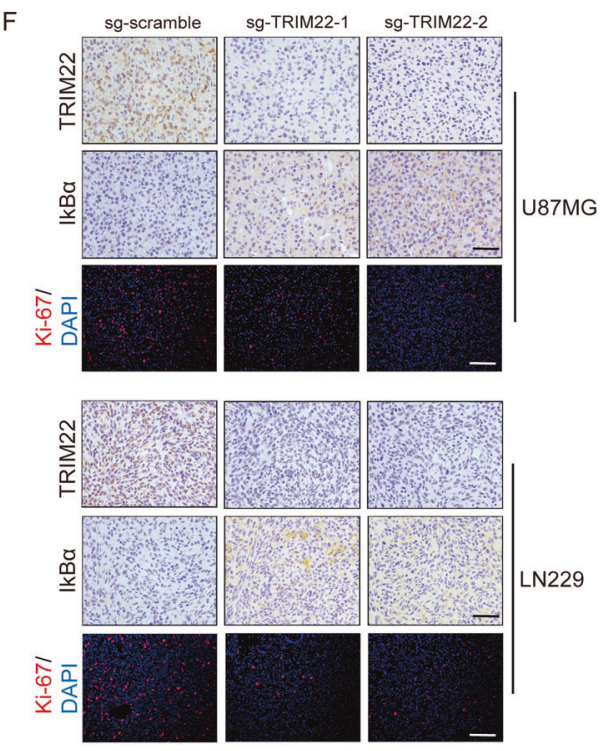

survival analysis performed with survival data from indicated groups. Log-rank test, $P<0.01$. f Representative images of IHC staining for TRIM22 and $\mathrm{I} \kappa \mathrm{B} \alpha$, and IF staining for Ki-67 levels in xenograft sections from NC and sg-TRIM22-1 and -2 groups. Scale bars, $50 \mu \mathrm{m}$ for IHC, and $100 \mu \mathrm{m}$ for Ki-67 IF staining. Student's $t$ test: n.s. not significant, $* P<0.05, * * P<0.01$.

47 days vs. 29 days, sg-TRIM22-1 or -2 vs. sg-scramble, respectively, $P<0.01$; LN229: 52 or 52 days vs. 34 days, sg-TRIM22-1 or -2 vs. sg-scramble, respectively, $P<$ 0.01; Fig. 2e). IHC staining performed on sections from xenografts showed that $\mathrm{I} \mathrm{B} \alpha$ expression levels increased in the absence of TRIM22, while Ki-67-IF staining was decreased (Fig. 2f and Supplementary Fig. S4A, B). 
Collectively, these data indicated that TRIM22 promoted growth of GBM tumors in vivo.

\section{E3 ligase activity is required for TRIM22-mediated GBM cell proliferation}

TRIM22 is a RING domain E3 Ub ligase mediating protein ubiquitination [31]. We therefore constructed two E3 ligase defective TRIM22 mutants, one with a RING domain deletion (TRIM22- $\Delta$ RING) and the other with the amino acid changes at conserved cysteines $\mathrm{C} 15$ and $\mathrm{C} 18$ to alanines (TRIM22-C15/18A) [32-34], to determine whether the growth-promoting activity of TRIM22 is associated with its E3 Ub ligase activity. Flag-tagged TRIM22- $\Delta$ RING, TRIM22-C15/18A, TRIM22-full length (TRIM22-FL), and empty vector (EV) were transfected into LN229 and U118MG. Phosphorylated IKK $\alpha / \beta$ (Ser176/180), ІкB $\alpha$ (Ser32/36) and P65 (Ser536) were elevated, while total I $\mathrm{B} \alpha$ was decreased, in cells overexpressing TRIM22 (TRIM22-FL) compared with EV groups (Fig. 3a and Supplementary Fig. S5A). However, each of these proteins remained unchanged in TRIM22- $\Delta$ RING/-C15/18A groups compared with EV groups (Fig. 3a and Supplementary Fig. S5A). TRIM22-FL also significantly increased cell proliferation (Fig. 3b, c and Supplementary Fig. S5B). In contrast, the RING domain deletion and C15/18A mutants did not enhance cell proliferation over controls (Fig. 3b, c and Supplementary Fig. S5B).

These data were confirmed in orthotopic xenograft models (Fig. 3d, e and Supplementary Fig. S5C, D). Survival time of tumor-bearing mice was shorter in LN229- and U118MG-TRIM22-FL groups (LN229: 26 vs. 39 days, TRIM22-FL vs. EV, respectively, $P<0.05$; U118MG: 27 vs. 34 days, TRIM22-FL vs. EV, respectively, $P<0.05$; Fig. 3f), but remained stable in LN229- and U118MG-TRIM22$\Delta$ RING (LN229: 39 vs. 39 days, TRIM22- $\Delta$ RING vs. $\mathrm{EV}$, respectively, $P=$ n.s.; U118MG: 35 vs. 34 days, TRIM22- $\Delta$ RING vs. EV, respectively, $P=$ n.s.; Fig. $3 f$ ) relative to EV groups. Taken together, these data suggested that the TRIM22 plays an important role in driving tumor growth possibly through its E3 Ub ligase activity.

\section{TRIM22 ubiquitinates IKBa}

To explore whether the E3 ligase activity is critical for TRIM22-mediated activation of NF- $\mathrm{KB}$ signaling, we tested TRIM22- $\Delta$ RING and TRIM22-C15/18A in NF- $\mathrm{BB}$ luciferase reporter assays compared with TRIM22-FL and EV groups. NF- $\mathrm{KB}$ signaling activity increased significantly in LN229 and U118MG cells transfected with TRIM22-FL, but remained unchanged in cells expressing TRIM22$\Delta$ RING/-C15/18A (Fig. 4a and Supplementary Fig. S5E). Elevated nuclear accumulation of P65 in LN229- and
U118MG-TRIM22-FL groups was the further demonstration of an enhanced activation of NF- $\mathrm{kB}$ signaling, but remained unchanged in cells expressing TRIM22- $\Delta$ RING/C15/18A (Fig. 4b and Supplementary Fig. S5F). In CHX studies, the half-life of IкB $\alpha$ was decreased by $\sim 4 \mathrm{~h}$ in LN229-TRIM22-FL cells and $\sim 8 \mathrm{~h}$ in LN229-TRIM22-FL cells compared to EV groups, while changes in LN229- and U118MG-TRIM22- $\Delta$ RING/-C15/18A were not significant (Fig. 4c, d and Supplementary Fig. S5G, H). Furthermore, both in vitro and in vivo, TRIM22-FL overexpression induced endogenous $\mathrm{K} 48$-linked ubiquitination of I $\mathrm{\kappa B} \alpha$, while the RING domain deletion and C15/18A mutants had no effect (Fig. 4e, f and Supplementary Fig. S5I).

To test whether TRIM22 directly binds to IкB $\alpha$, we performed co-IPs using lysates prepared from HEK293 transiently transfected with Flag-tagged TRIM22 and HA-tagged I $\mathrm{B} \alpha$ expression vectors. Immunoprecipitation with anti-FLAG or anti-HA antibodies brought down both Flag-tagged TRIM22 and HA-tagged I $\mathrm{BB} \alpha$ indicating that the two tagged proteins were associated with each other in HEK293 cells (Fig. 4g). An endogenous physical interaction between the two proteins was confirmed in coIPs using U87MG, LN229, and U118MG cell lysates (Fig. 4h). To investigate the functional domains responsible for their interaction, a series of deletion mutant constructs for TRIM 22 and IкB $\alpha$ were expressed in cells, and co-IPs were performed. AR4 and AR5 domains in IкB $\alpha$, or amino acids $182-317$, brought down TRIM22. In TRIM22, amino acids 89-131 (B-Box domain), 133-223 (coiled-coil domain), and 352-498 (SPRY domain) were necessary to bring down IкB $\alpha$ (Fig. 4i, j). Interestingly, although the TRIM22 ring domain mutant was less efficient at binding I $\mathrm{K} \mathrm{B} \alpha$, the domain was not essential for the two proteins to interact. Thus, TRIM22 might promote the development of human glioma by facilitating proteasomal-mediated degradation of $І \kappa \mathrm{B} \alpha$, a negative regulator of $\mathrm{NF}-\mathrm{KB}$ signaling.

\section{TRIM22 promotes K63-linked ubiquitination of IKKץ}

Loss of TRIM22 also led to decreased phosphorylation of $\mathrm{IKK} \alpha / \beta$ (Ser176/180), IкB $\alpha$ (Ser32/36), and P65, indicating that it may have some role in the phosphorylation and thus activation of these proteins, which are mediators of so-called canonical NF-kB signaling (Fig. 1b). We therefore performed co-IP assays with anti-TRIM22 antibodies to determine whether any of these proteins were associated with TRIM22 in GBM cell lines. We found that IKK $\gamma$, rather than IKK $\alpha / \beta$, was associated with TRIM22 in GBM cells (Fig. 5a). We furthermore found that K63 ubiquitination levels of IKK $\gamma$ paralleled TRIM22 protein levels (Fig. 5b). In summary, these data suggested that TRIM22 might regulate $\mathrm{NF}-\kappa \mathrm{B}$ activation through activation of the IKK complex. 
A

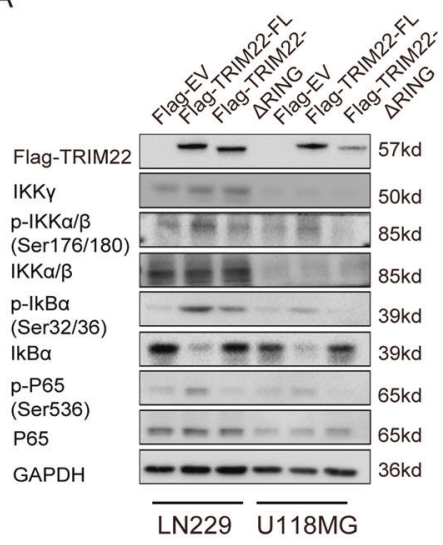

D
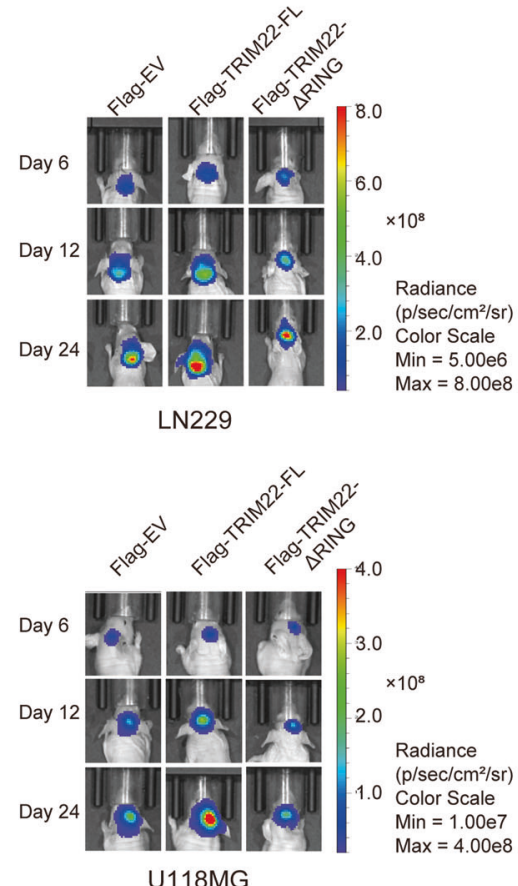

B

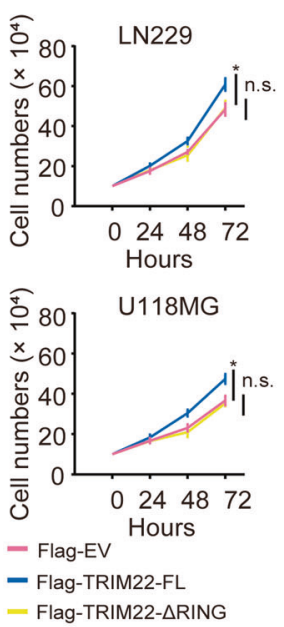

C

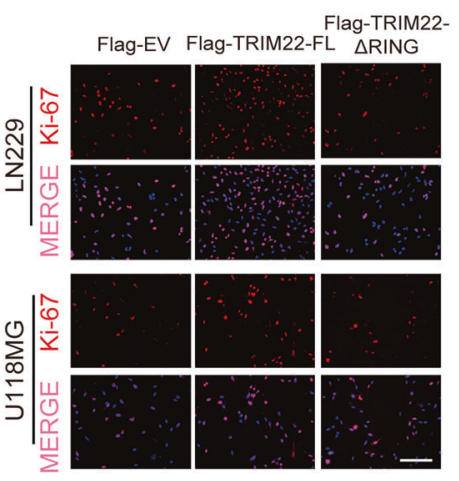

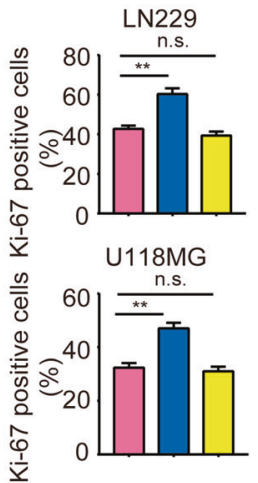

- Flag-EV

- Flag-TRIM22-FL

- Flag-TRIM22- - RING

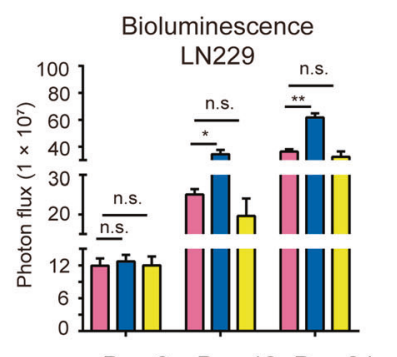

Day 6 Day 12 Day 24 Days after tumor implantation

- Flag-EV

Elag-TRIM22-FL

Flag-TRIM22- $\triangle$ RING

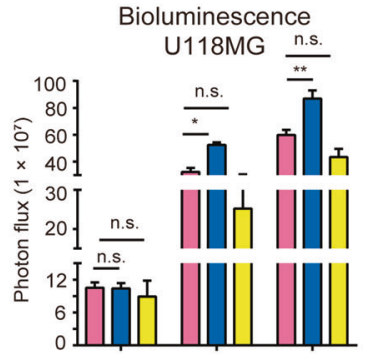

Day 6 Day 12 Day 24 Days after tumor implantation

$$
\begin{aligned}
& \text { Flag-EV } \\
& \text { Flag-TRIM22-FL } \\
& \text { Flag-TRIM22- } \triangle \text { RING }
\end{aligned}
$$

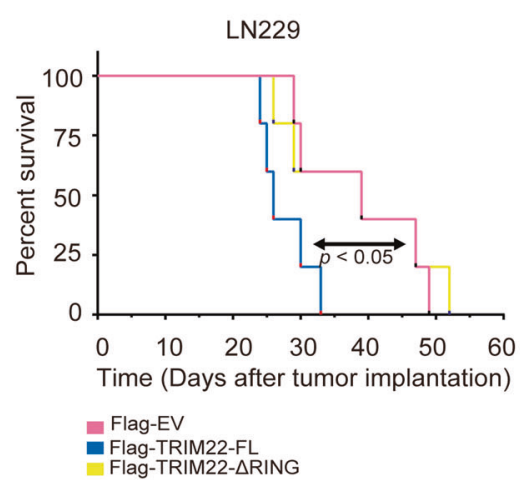

U118MG

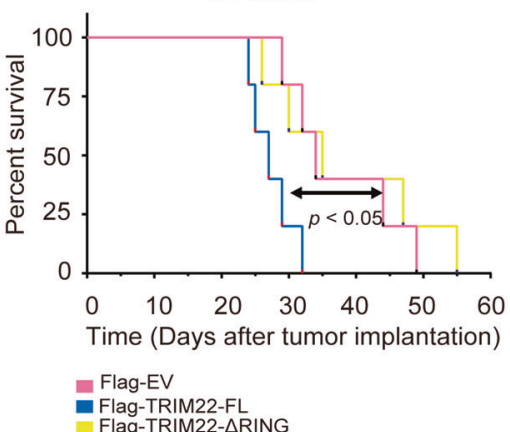

Fig. 3 Overexpression of TRIM22 promotes proliferation of GBM in vitro and in vivo via its RING domain. a Western blot analysis to evaluate components of the NF- $\mathrm{KB}$ pathway in lysates prepared from LN229- and U118MG-EV, TRIM22-FL and TRIM22- $\Delta$ RING cell populations. GAPDH was used as the loading control. b Growth curves generated using cell counting and $\mathbf{c} \mathrm{Ki}-67$ immunofluorescence staining for transfected cells in a. Scale bars, $100 \mu \mathrm{m}$. d, e

\section{IKBa mediates the tumor growth-promoting effects of TRIM22}

To determine whether TRIM22 activates NF- $\mathrm{B}$ signaling through $\mathrm{I} \kappa \mathrm{B} \alpha$, we introduced a construct expressing an I $\mathrm{B} \mathrm{B} \alpha$
Representative images and quantification of in vivo luciferase bioluminescence from indicated cells orthotopically implanted into the brains of nude mice at day 6, 12, and 24 after injection. f Kaplan-Meier analysis of survival for tumor-bearing mice implanted with indicated cells. Log-rank test, $P<0.05$. Student's $t$ test: n.s. not significant, $* P<0.05, * * P<0.01$.

phosphorylation mutant (S32/36A; srIkB $\alpha$ ) into LN229 and U118MG cells. The substitution of S32 and S36 with alanine leads to a constitutively unphosphorylated state in $\operatorname{srI} \kappa \mathrm{B} \alpha$, which prevents its degradation and subsequent NF- $\kappa \mathrm{B}$ activation [35]. Thus, we first tested whether TRIM22 induced 
A

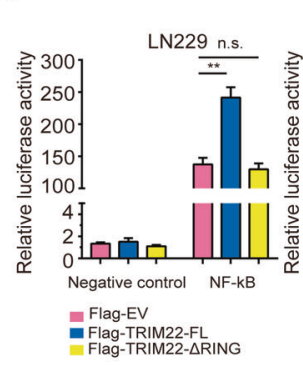

C

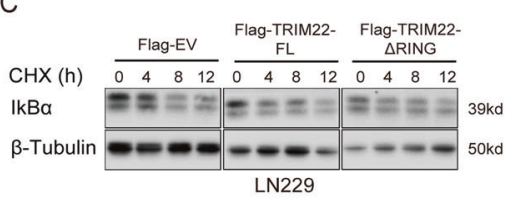

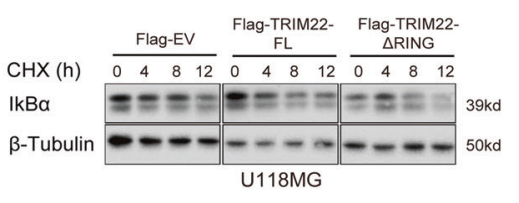

$\mathrm{F}$
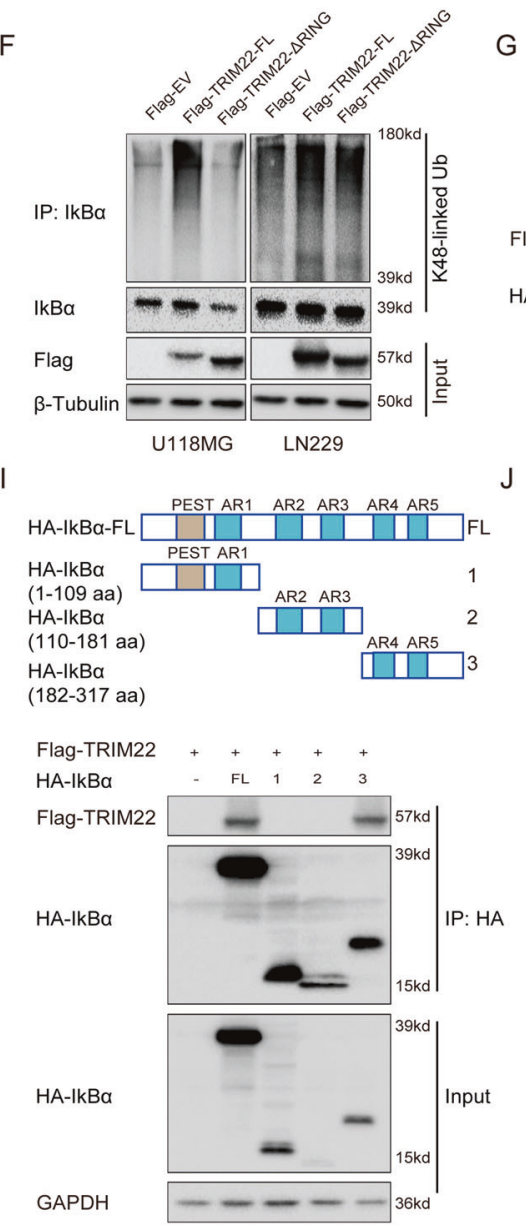

G
B
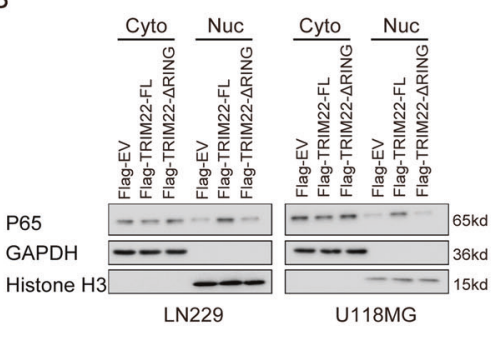

D
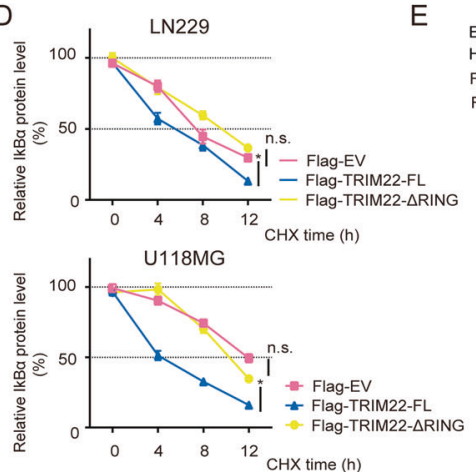

$\mathrm{H}$

$E$

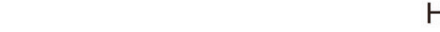

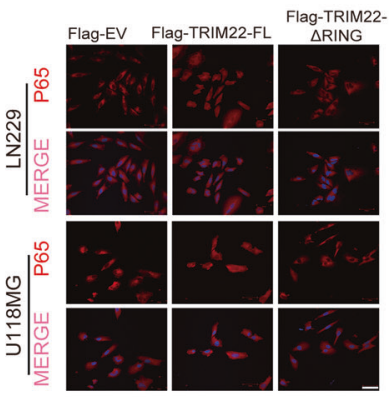
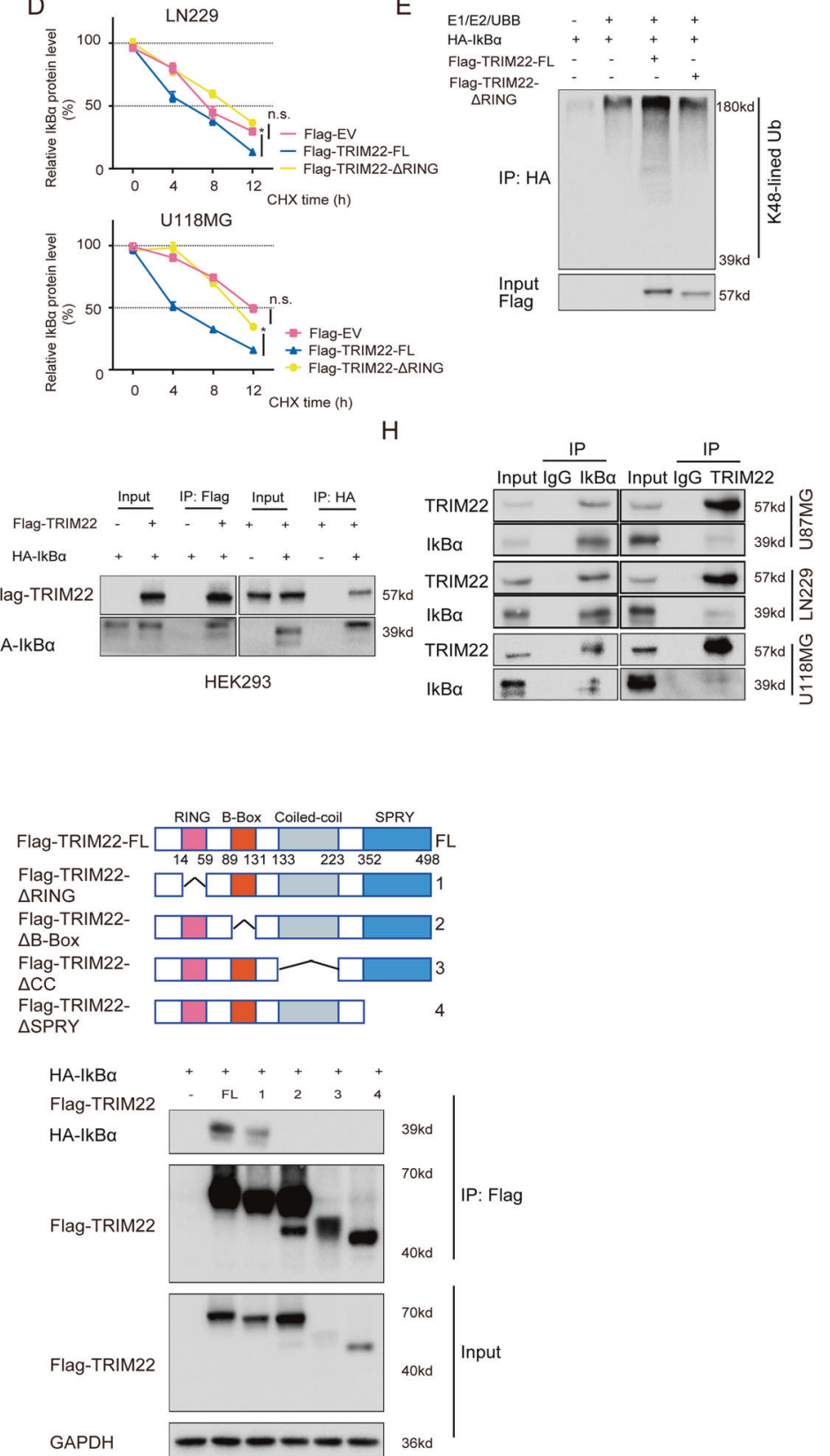

$\mathrm{NF}-\kappa \mathrm{B}$ activity in the presence of $\operatorname{srI} \kappa \mathrm{B} \alpha$ in glioma cells, using the NF- $\mathrm{KB}$ luciferase reporter construct. In LN229- and U118MG-TRIM22-FL cells, NF- $\kappa B$ transcriptional activity was increased relative to controls $(\sim 2 \times$; Fig. 6a). However, NF- $\kappa \mathrm{B}$ activity did not increase in TRIM22 overexpressing cells in the presence of srIкB $\alpha$ (Fig. 6a). 
Fig. 4 TRIM22 ubiquitinates IкB $\boldsymbol{\alpha}$. a Luciferase activity for NF- $\mathrm{NB}$ luciferase or control reporter constructs in modified LN229 and U118MG cells. b Western blot analysis of cytoplasmic (Cyto) and nuclear (Nuc) fractions prepared from indicated cells. Immunofluorescence for P65 in modified LN229 and U118MG cells showing cellular localization. Scale bars, $20 \mu \mathrm{m}$. c Western blot to detect IкB $\alpha$ levels after $0,4,8$, and $12 \mathrm{~h}$ of cycloheximide (CHX; $25 \mu \mathrm{g} / \mathrm{mL}$ ) treatment in modified LN229 and U118MG cells compared with controls. d Line graph showing IкB $\alpha$ protein levels normalized to $\beta$ tubulin and to $0 \mathrm{~h}$ at the indicated time points. e Western blot of IP of $\mathrm{I} \kappa \mathrm{B} \alpha$ incubated with anti-K48-linkage specific polyubiquitin antibody to detect ubiquitination of $\mathrm{I} \kappa \mathrm{B} \alpha$ in an in vitro assay. $\mathrm{f}$ In vivo ubiquitination assay of IкB $\alpha$. $\mathbf{g}$ Western blot analysis of co-IPs performed on lysates prepared from HEK293 cells transfected with Flag-TRIM22 and HA-I $\mathrm{HB} \alpha$. h Western blot analysis of co-IPs performed using antiIкB $\alpha$ or -TRIM22 antibody on lysates prepared from U87MG, LN229, and U118MG cells. i Schematic representation of wild-type IкB $\alpha$ and the indicated deletion mutants. Western blot analysis of co-IPs performed on lysates prepared from HEK293 cells transfected with FlagTRIM22 alone or together with indicated HA-IкB $\alpha$ constructs. Upper panels represent co-IPs performed with anti-HA; lower panels represent input protein. $\mathbf{j}$ Schematic representation of wild-type TRIM22 and the indicated deletion mutants. Western blot analysis of co-IPs performed on lysates prepared from HEK293 cells transfected with HA-IкB $\alpha$ alone or together with indicated Flag-TRIM22 constructs. Student's $t$ test: n.s. not significant, ${ }^{*} P<0.05, * * P<0.01$.

In cell number counting assays, $\operatorname{srI} \kappa \mathrm{B} \alpha$ blocked the increase in cell proliferation mediated by overexpression of TRIM22 in LN229 and U118MG cells relative to controls (Fig. 6b). The growth-promoting properties of TRIM22 were also shown to be mediated by $\mathrm{I} \kappa \mathrm{B} \alpha$ in an orthotopic tumor model. LN229- and U118MG-TRIM22-FL tumors were greater in size compared with EV groups, but this effect was abolished by $\operatorname{srI} \kappa \mathrm{B} \alpha$ overexpression (Fig. 6c, d). Overall survival was also correspondingly reduced in mice bearing LN229- and U118MG-TRIM22-FL tumors relative to control mice (LN229: 27 vs. 35 days, TRIM22-FL vs. EV, respectively, $P<0.05$; U118MG: 29 vs. 39 days, TRIM22-FL vs $\mathrm{EV}$, respectively, $P<0.05$; Fig. 6e). However, srI $\kappa \mathrm{B} \alpha$ overexpression in the context of TRIM22 overexpression brought overall survival back to nearly control group levels (LN229: 35 vs. 39 days, TRIM22-FL $+\operatorname{srI} \kappa B \alpha$ vs. EV, respectively, $P=$ n.s.; U118MG: 35 vs. 35 days, TRIM22-FL $+\operatorname{srI\kappa B} \alpha$ vs. $\mathrm{EV}$, respectively, $P=$ n.s.; Fig. 6e). These results indicated that $\mathrm{I} \kappa \mathrm{B} \alpha$ is a key effector in TRIM22-promoted growth of GBM cell populations in vitro and in vivo.

\section{TRIM22 predicts higher grade of human glioma malignancy}

To characterize its role in the progression of human gliomas, we evaluated TRIM22 protein levels in a cohort of primary gliomas on a tissue microarray and NBT samples using IHC. The histology and distribution of the samples was as follows: WHO grade II $(n=30)$, WHO grade III $(n=31)$, WHO grade IV $(n=51, \mathrm{GBMs})$, and nonneoplastic brain (NBT, $n=10$ ). TRIM22 was preferentially
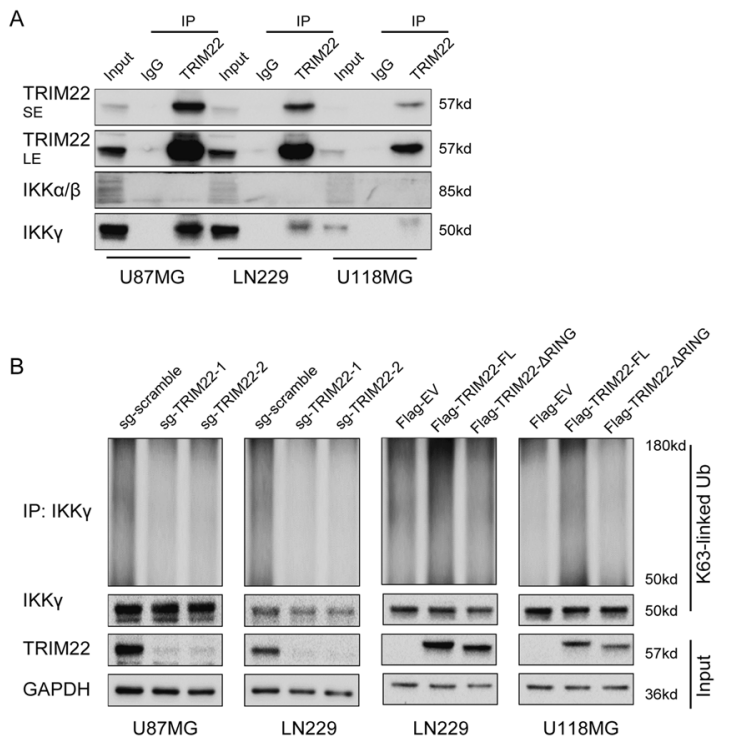

Fig. 5 TRIM22 promotes K63-linked ubiquitination of IKK $\gamma$. a Western blot analysis of co-IPs to demonstrate association of TRIM22 with IKK $\alpha / \beta$ or IKK $\gamma$ in U87MG, LN229, and U118MG cells. b Western blot analysis for IKK $\gamma$ of co-IPs with K63-linkage specific polyubiquitin antibody in the indicated modified U87MG, LN229, and U118MG cells.

expressed in high grade gliomas $(n=82$; HGG, WHO grade III-IV) compared with low grade gliomas $(n=30$; LGG, WHO grade II; $P<0.001$; Fig. $7 \mathrm{a}, \mathrm{b}$ and Supplementary Table S4). Expression in NBT samples was nearly absent (Fig. 7a, b and Supplementary Fig. S6A). WB of lysates prepared from primary tumors $(n=12$; WHO grades II-IV) and NBT samples $(n=3)$ confirmed the overexpression of TRIM22 in human gliomas (Fig. 7c). Finally, increased expression of TRIM22 correlated with other features of more aggressive gliomas, including wild-type IDH1 $(P<0.001)$ and wild-type ATRX $(P=0.0054)$.

We also performed experiments with GBM\#P3 and GSC\#BG5 cells, which more faithfully retain the genetic features of the matching primary GBM [36]. Knockout of TRIM22 in GBM\#P3 cells led to decreased tumor growth in vivo (Fig. 7d, e) and prolonged the survival time of tumor-bearing mice (GBM\#P3: 44 or 47 days vs. 34 days, sg-TRIM22-1 or -2 vs. sg-scramble, respectively, $P<0.05$; BG5: 55 or 52 days vs. 35 days, sg-TRIM22-1 or 2 vs. sg-scramble, respectively, $P<0.05$; Fig. 7f). Thesedata are in agreement with a growth-promoting role for TRIM22 in the development of human gliomas.

\section{Discussion}

Targeting NF- $\mathrm{KB}$ signaling is emerging as a promising therapeutic strategy for GBM. To date, studies regarding $\mathrm{NF}-\kappa \mathrm{B}$ as a potential therapeutic target have focused on 
A

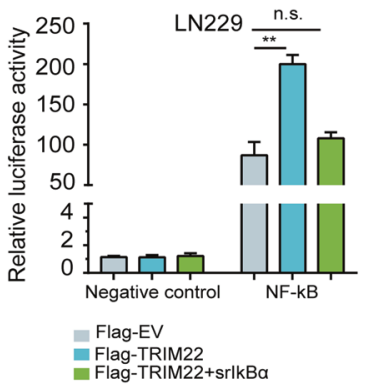

C

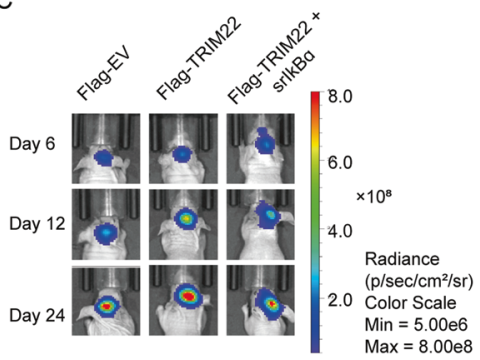

LN229

E

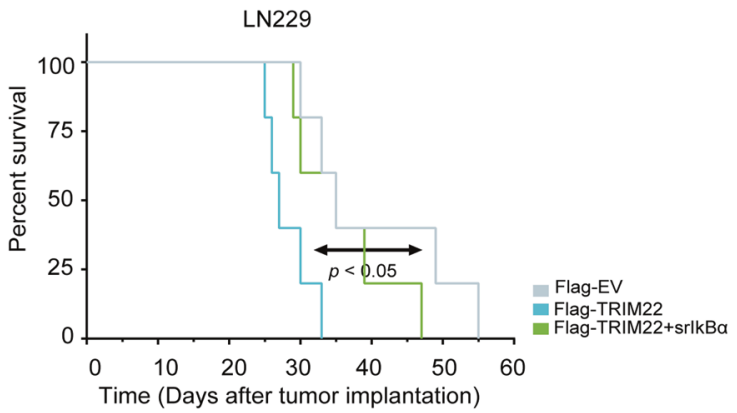

Fig. 6 TRIM22 promotes the growth of GBM via IкB $\alpha$. a Luciferase activity in modified LN229 and U118MG cells transfected with NF- $\kappa B$ luciferase reporter constructs. b Growth curves generated with cell counting performed over $72 \mathrm{~h}$. c, d In vivo bioluminescence imaging and quantification of modified LN229 and U118MG cells

nonspecific compounds or inhibitors of IKK, which impact many of the pathways central to the malignant phenotype of GBM [37]. However, NF-אB inhibitors initiate a broad range of responses and side effects. Thus, glioma patients may benefit from inhibition of specific factors upstream of various mediators of NF- $\mathrm{KB}$ signaling [38]. In this study, we identified TRIM22 as a potential modulator of NF- $\mathrm{KB}$ signaling, by screening activity from an NF-kB-dependent transcriptional luciferase reporter construct in response to knockout of five TRIM genes overexpressed in GBM. We demonstrated that TRIM22 promotes glioma cell proliferation in vitro and tumor formation in vivo, and that these properties are linked to its intrinsic E3 Ub ligase activity. TRIM22 aids in the generation of K48-linked Ub conjugates
B
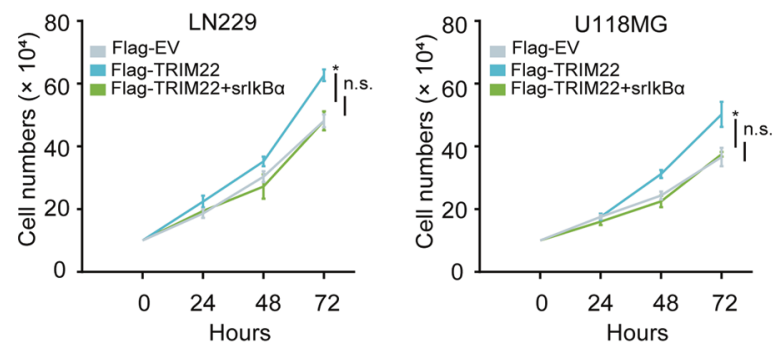

D
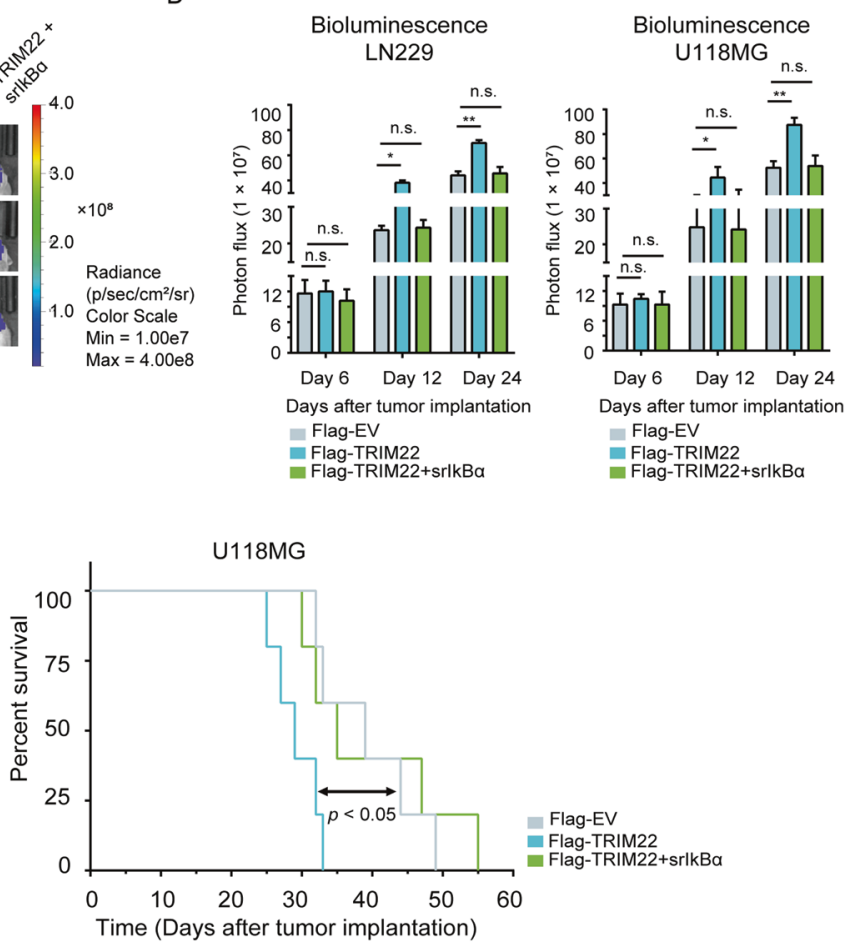

derived xenografts at the indicated time points. e Kaplan-Meier survival analysis performed with survival data of tumor-bearing animals implanted with the indicated cells. Log-rank test, $P=$ n.s., $P<0.05$. Student's $t$ test: n.s. not significant, $* P<0.05,{ }^{*} P P<0.01$.

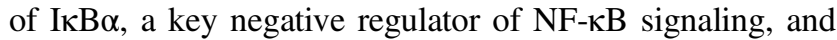
thus promotes enhanced proteasomal-mediated degradation of the protein. TRIM22 also associates and generates K63linked $\mathrm{Ub}$ conjugates of IKK $\gamma$, which in turn activate IKK complexes and subsequently degrade I $\mathrm{KB} \alpha$ (model in Fig. 7g). TRIM22 exhibited increased expression in primary human GBM specimens, and high TRIM22 correlated with factors associated with higher grade gliomas. Our study therefore revealed a novel regulatory mechanism for I $\mathrm{I} B \alpha$ degradation and NF- $\mathrm{KB}$ activation, and a critical role for TRIM22 in glioma tumorigenesis.

TRIM22 was first identified as an IFN-induced protein and also found to be a transcriptional target gene of TP53 $[39,40]$. Although the proximal promoter of TRIM22 is not 
A

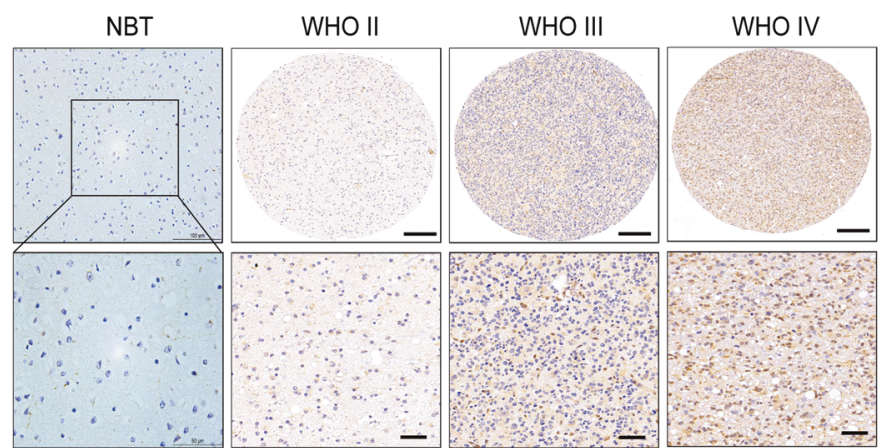

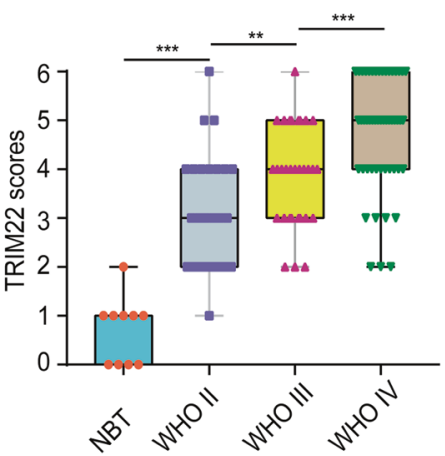

C

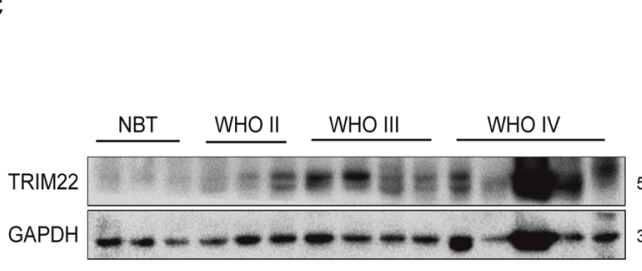

D

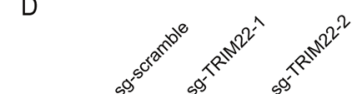
57kd

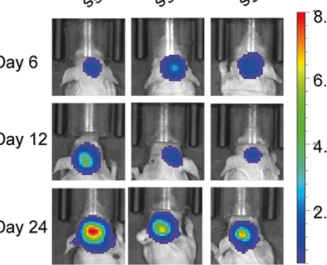

GBM\#P3

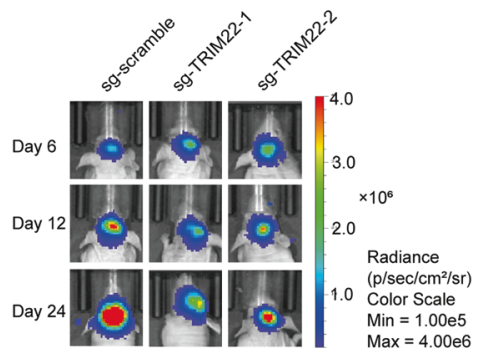

BG5

E
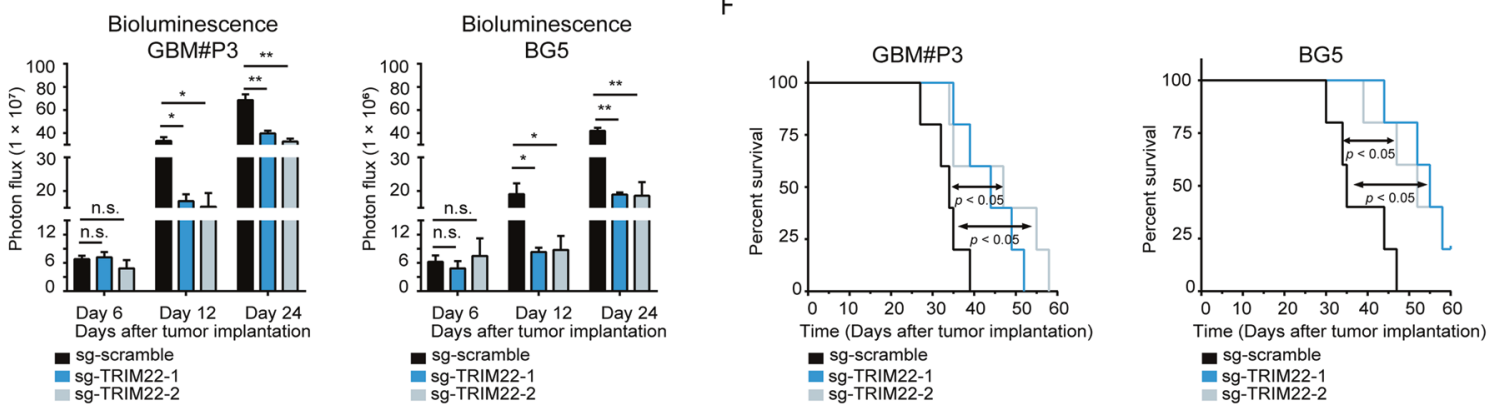

G

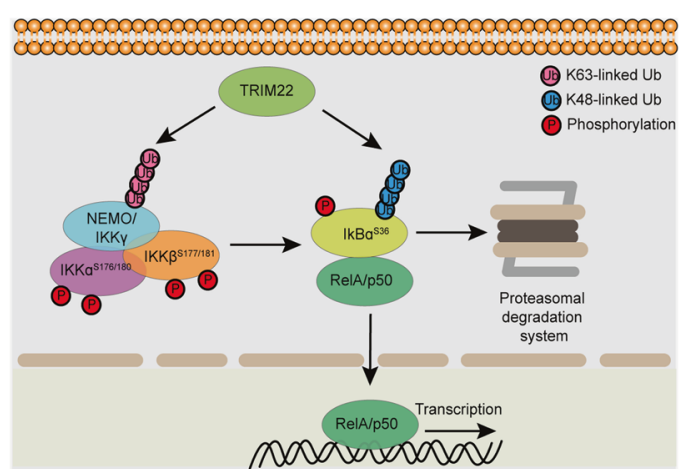

p53-responsive, a functional enhancer-like element was found in intron 1 of the TRIM22 gene. TP53, which is frequently deregulated in GBM, plays a central role in the classification of GBM molecular subtypes and GBM progression [41]. We found that TRIM22 is preferentially expressed in U87MG and A172 cell lines (P53 wild type) compared with LN229 and U118MG cell lines (P53 mutated; data not shown here). The significance of the association between TRIM22 and P53 status in human glioma requires further investigation.

TRIM22 contains a conserved RING domain, which suggests that it has a potential role as an E3 Ub ligase 
Fig. 7 TRIM22 is highly expressed in GBM samples and correlates with other clinical parameters associated with higher grade glioma. a Images of TRIM22 IHC performed on WHO grade II-IV glioma tissue microarrays and nonneoplastic brain tissues (NBT). Representative image of NBT was from the frontal lobe. Scale bar = $100 \mu \mathrm{m}$ (top) or $50 \mu \mathrm{m}$ (bottom). For glioma tissue microarrays, scale bar $=200 \mu \mathrm{m}$ (top) or $50 \mu \mathrm{m}$ (bottom). b Graphic representation of scoring performed on IHC staining for TRIM22 in primary samples from glioma tissue microarrays and NBTs. $\mathbf{c}$ Western blot analysis of TRIM22 protein levels in primary glioma tissues $(n=12)$ and nonneoplastic brain tissue samples $(n=3)$. d, e In vivo bioluminescence imaging and quantification of modified GBM\#P3 and GSC\#BG5 cells derived xenografts at the indicated time points. f Kaplan-Meier survival analysis performed with survival data from indicated cells. Logrank test, $P<0.05$. g A graphical model for TRIM22-mediated NF- $\mathrm{kB}$ activation and GBM growth, showing interaction of TRIM22 with critical components of the pathway, including $\mathrm{I} \kappa \mathrm{B} \alpha$ which normally sequesters the transcription activating complex in the cytoplasm. TRIM22 targets I $\mathrm{KB} \alpha$ for proteasomal-mediated degradation and activates the IKK complex through K63-linked ubiquitination of IKK $\gamma / \mathrm{NEMO}$, which leads to further disruption of IкB $\alpha$ from the complex. Student's $t$ test: n.s. not significant, $* P<0.05$, $* * P<0.01$, $* * * P<0.001$.

involved in posttranscriptional modification of certain proteins $[31,32,42]$. Specificity of the Ub conjugation system is derived from the direct association of the E3 ligase with its substrates. Here, we demonstrated that TRIM22 interacted with both IKB $\alpha$ and IKK $\gamma$, causing ubiquitination which was E3 ligase activity-dependent. Overexpression of TRIM22, which promoted glioma cell proliferation in vivo and in vitro, was blocked through deletion of the RING domain or substitution of two conserved cysteine sites by alanine. Thus, we determined that the E3 ligase activity of TRIM22 was required for its function. However, it has been reported that the C terminal SPRY domain [27, 32, 43], which is in a region that we found to be necessary for the interaction between TRIM 22 and I $\mathrm{KB} \alpha$, is also important for TRIM22-mediated biological functions. Therefore, further study is necessary to determine which region of TRIM22 might be relevant to the development of human gliomas.

IKK $\gamma$, also known as the NF- $\mathrm{KB}$ essential modulator, is a regulatory subunit of the IKB kinase complex and has a critical role in the activation of NF- $\kappa \mathrm{B}$ [44]. I $\kappa \mathrm{B} \alpha$ binds strongly to NF- $\mathrm{kB}$ and sequesters it in the cytoplasm in resting cells. Upon stimulation, the IкB kinase/IKK complex phosphorylates $\mathrm{I} \kappa \mathrm{B} \alpha$ at serine $32 / 36$. NF- $\kappa \mathrm{B}$ becomes active as phosphorylated $\mathrm{I} \kappa \mathrm{B} \alpha$ is released, and undergoes K48-linked polyubiquitination and proteasome-dependent degradation [37]. Previous studies have demonstrated that the F-box protein $\beta$-TrCP1 is involved in the proteasomalmediated degradation of $\mathrm{I} \kappa \mathrm{B} \alpha$ [45]. However, a subsequent study showed that $\mathrm{I} \kappa \mathrm{B} \alpha$ was still degraded when $\beta$-TrCP1 was knocked out, suggesting that other E3 ligases might target $\mathrm{I} \kappa \mathrm{B} \alpha$ for proteasomal-mediated degradation [45]. Therefore, we screened the activity of a panel of TRIM proteins using the NF- $\mathrm{KB}$ response reporter, which led to the identification of TRIM22 as a potential regulator of NF$\kappa \mathrm{B}$ signaling. We demonstrated that TRIM22-targeted IкB $\alpha$ for proteasomal-mediated degradation and further enhanced this protein degradation by modifying K63-linked polyubiquitin chains on IKK $\gamma$, despite the existence of linear and K27-linked ubiquitination of IKK $\gamma$ [46, 47].

In summary, our study revealed a novel regulatory mechanism of NF- $\kappa$ B activation, in which TRIM22, an E3 ligase, promotes $\mathrm{K} 48$ - and $\mathrm{K} 63$-linked ubiquitination of

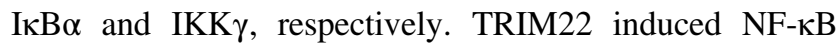
signaling in GBM, which drives tumor growth and progression. Finally, our study defines TRIM22 as a candidate therapeutic target. Pharmaceutical inhibition of its E3 ligase activity or the interaction between these three proteins may provide a promising strategy for the treatment of GBM.

Acknowledgements This work was supported by the National Natural Science Foundation of China (81972351, 81903126, 81701329, 81874083 , and 81702474), the Department of Science and Technology of Shandong Province (2017CXGC1502 and 2018CXGC1503), the Special Foundation for Taishan Scholars (ts20110814, tshw201502056, tsqn20161067, and tsqn201909173), the China Postdoctoral Science Foundation (2018M642666), the Jinan Science and Technology Bureau of Shandong Province (2019GXRC006), the Norwegian Research Council, The Norwegian Cancer Society and Helse-Vest, Haukeland University Hospital, and the Shandong Research Institute of Industrial Technology.

Author contributions JXJ, KKD, BH, XGL, and JW conceived and designed the project. JXJ and KKD performed experiments. JXJ, $\mathrm{KKD}, \mathrm{TL}$, and $\mathrm{XZ}$ analyzed the data. JXJ, KKD, and JW wrote the manuscript. AJC and DZ provided reagents and materials. FT gave intellectual input. BH, XGL, and JW supervised the study.

\section{Compliance with ethical standards}

Conflict of interest The authors declare that they have no conflict of interest.

Publisher's note Springer Nature remains neutral with regard to jurisdictional claims in published maps and institutional affiliations.

Open Access This article is licensed under a Creative Commons Attribution 4.0 International License, which permits use, sharing, adaptation, distribution and reproduction in any medium or format, as long as you give appropriate credit to the original author(s) and the source, provide a link to the Creative Commons license, and indicate if changes were made. The images or other third party material in this article are included in the article's Creative Commons license, unless indicated otherwise in a credit line to the material. If material is not included in the article's Creative Commons license and your intended use is not permitted by statutory regulation or exceeds the permitted use, you will need to obtain permission directly from the copyright holder. To view a copy of this license, visit http://creativecommons. org/licenses/by/4.0/.

\section{References}

1. Rayet B, Gelinas C. Aberrant rel/nfkb genes and activity in human cancer. Oncogene. 1999;18:6938-47. 
2. Naugler WE, Karin M. NF-kappaB and cancer-identifying targets and mechanisms. Curr Opin Genet Dev. 2008;18:19-26.

3. Karin M, Greten FR. NF-kappaB: linking inflammation and immunity to cancer development and progression. Nat Rev Immunol. 2005;5:749-59.

4. Bhat KPL, Balasubramaniyan V, Vaillant B, Ezhilarasan R, Hummelink K, Hollingsworth F, et al. Mesenchymal differentiation mediated by NF-kappaB promotes radiation resistance in glioblastoma. Cancer Cell. 2013;24:331-46.

5. Karin M. Nuclear factor-kappaB in cancer development and progression. Nature. 2006;441:431-6.

6. Hayden MS, Ghosh S. Shared principles in NF-kappaB signaling. Cell. 2008;132:344-62.

7. Raychaudhuri B, Han Y, Lu T, Vogelbaum MA. Aberrant constitutive activation of nuclear factor kappaB in glioblastoma multiforme drives invasive phenotype. J Neurooncol. 2007;85:39-47.

8. Wang $\mathrm{H}$, Wang $\mathrm{H}$, Zhang $\mathrm{W}$, Huang HJ, Liao WS, Fuller GN. Analysis of the activation status of Akt, NFkappaB, and Stat3 in human diffuse gliomas. Lab Invest. 2004;84:941-51.

9. Song L, Liu L, Wu Z, Li Y, Ying Z, Lin C, et al. TGF-beta induces miR-182 to sustain NF-kappaB activation in glioma subsets. J Clin Invest. 2012;122:3563-78.

10. Nagai S, Washiyama K, Kurimoto M, Takaku A, Endo S, Kumanishi T. Aberrant nuclear factor-kappaB activity and its participation in the growth of human malignant astrocytoma. J Neurosurg. 2002;96:909-17.

11. Verhaak RG, Hoadley KA, Purdom E, Wang V, Qi Y, Wilkerson $\mathrm{MD}$, et al. Integrated genomic analysis identifies clinically relevant subtypes of glioblastoma characterized by abnormalities in PDGFRA, IDH1, EGFR, and NF1. Cancer Cell. 2010;17:98-110.

12. Shi D, Grossman SR. Ubiquitin becomes ubiquitous in cancer: emerging roles of ubiquitin ligases and deubiquitinases in tumorigenesis and as therapeutic targets. Cancer Biol Ther. 2010;10:737-47.

13. Hatakeyama S. TRIM proteins and cancer. Nat Rev Cancer. 2011;11:792-804.

14. Akutsu M, Dikic I, Bremm A. Ubiquitin chain diversity at a glance. J Cell Sci. 2016;129:875-80

15. Swatek KN, Komander D. Ubiquitin modifications. Cell Res. 2016;26:399-422.

16. Liu S, Chen ZJ. Expanding role of ubiquitination in NF-kappaB signaling. Cell Res. 2011;21:6-21.

17. Chen ZJ. Ubiquitination in signaling to and activation of IKK. Immunol Rev. 2012;246:95-106.

18. Ea CK, Deng L, Xia ZP, Pineda G, Chen ZJ. Activation of IKK by TNFalpha requires site-specific ubiquitination of RIP1 and polyubiquitin binding by NEMO. Mol Cell. 2006;22:245-57.

19. Wang C, Deng L, Hong M, Akkaraju GR, Inoue J, Chen ZJ. TAK1 is a ubiquitin-dependent kinase of MKK and IKK. Nature. 2001;412:346-51.

20. Deng L, Wang C, Spencer E, Yang L, Braun A, You J, et al. Activation of the IkappaB kinase complex by TRAF6 requires a dimeric ubiquitin-conjugating enzyme complex and a unique polyubiquitin chain. Cell. 2000;103:351-61.

21. Chen ZJ, Parent L, Maniatis T. Site-specific phosphorylation of IkappaBalpha by a novel ubiquitination-dependent protein kinase activity. Cell. 1996;84:853-62.

22. Noguchi K, Okumura F, Takahashi N, Kataoka A, Kamiyama T, Todo $\mathrm{S}$, et al. TRIM40 promotes neddylation of IKKgamma and is downregulated in gastrointestinal cancers. Carcinogenesis. 2011;32:995-1004.

23. Lv D, Li Y, Zhang W, Alvarez AA, Song L, Tang J, et al. TRIM24 is an oncogenic transcriptional co-activator of STAT3 in glioblastoma. Nat Commun. 2017;8:1454.
24. Yu S, Gao B, Duan Z, Xu W, Xiong S. Identification of tripartite motif-containing 22 (TRIM22) as a novel NF-kappaB activator. Biochem Biophys Res Commun. 2011;410:247-51.

25. Lou J, Wang Y, Zheng X, Qiu W. TRIM22 regulates macrophage autophagy and enhances Mycobacterium tuberculosis clearance by targeting the nuclear factor-multiplicity kappaB/beclin 1 pathway. J Cell Biochem. 2018;119:8971-80.

26. Kang C, Lu Z, Zhu G, Chen Y, Wu Y. Knockdown of TRIM22 relieves oxygen-glucose deprivation/reoxygenation-induced apoptosis and inflammation through inhibition of NF-kappaB/ NLRP3 Axis. Cell Mol Neurobiol. 2020. https://doi.org/10.1007/ s10571-020-00855-w. [Online ahead of print].

27. Qiu H, Huang F, Gong J, Xiao H, Sun BL, Yang RG. TRIM22 can activate the noncanonical NF-kappaB pathway by affecting IKKalpha. J Recept Signal Transduct Res. 2015;35:289-94.

28. Qiu H, Huang F, Xiao H, Sun B, Yang R. TRIM22 inhibits the TRAF6-stimulated NF-kappaB pathway by targeting TAB2 for degradation. Virol Sin. 2013;28:209-15.

29. Ji J, Xu R, Ding K, Bao G, Zhang X, Huang B, et al. Long noncoding RNA SChLAP1 forms a growth-promoting complex with HNRNPL in human glioblastoma through stabilization of ACTN4 and activation of NF-kappaB signaling. Clin Cancer Res. 2019;25:6868-81.

30. Ding K, Ji J, Zhang X, Huang B, Chen A, Zhang D, et al. RNA splicing factor USP39 promotes glioma progression by inducing TAZ mRNA maturation. Oncogene. 2019;38:6414-28.

31. Duan Z, Gao B, Xu W, Xiong S. Identification of TRIM 22 as a RING finger E3 ubiquitin ligase. Biochem Biophys Res Commun. 2008;374:502-6.

32. Gao B, Duan Z, Xu W, Xiong S. Tripartite motif-containing 22 inhibits the activity of hepatitis B virus core promoter, which is dependent on nuclear-located RING domain. Hepatology. 2009;50:424-33.

33. Barr SD, Smiley JR, Bushman FD. The interferon response inhibits HIV particle production by induction of TRIM22. PLoS Pathog. 2008;4:e1000007.

34. Kajaste-Rudnitski A, Marelli SS, Pultrone C, Pertel T, Uchil PD, Mechti N, et al. TRIM22 inhibits HIV-1 transcription independently of its E3 ubiquitin ligase activity, Tat, and NF-kappaBresponsive long terminal repeat elements. J Virol. 2011;85:5183-96.

35. Traenckner EB, Pahl HL, Henkel T, Schmidt KN, Wilk S, Baeuerle PA. Phosphorylation of human I kappa B-alpha on serines 32 and 36 controls I kappa B-alpha proteolysis and NFkappa B activation in response to diverse stimuli. EMBO J. 1995; 14:2876-83

36. da Hora CC, Schweiger MW, Wurdinger T, Tannous BA. Patientderived glioma models: from patients to dish to animals. Cells. 2019;8:1177.

37. Cahill KE, Morshed RA, Yamini B. Nuclear factor-kappaB in glioblastoma: insights into regulators and targeted therapy. Neuro Oncol. 2016;18:329-39.

38. Mansour NM, Bernal GM, Wu L, Crawley CD, Cahill KE, Voce DJ, et al. Decoy receptor DcR1 is induced in a p50/Bcl3-dependent manner and attenuates the efficacy of temozolomide. Cancer Res. 2015;75:2039-48.

39. Gongora C, Tissot C, Cerdan C, Mechti N. The interferon-inducible Staf50 gene is downregulated during $\mathrm{T}$ cell costimulation by $\mathrm{CD} 2$ and CD28. J Interferon Cytokine Res. 2000;20:955-61.

40. Obad S, Brunnstrom H, Vallon-Christersson J, Borg A, Drott K, Gullberg U. Staf50 is a novel p53 target gene conferring reduced clonogenic growth of leukemic U-937 cells. Oncogene. 2004;23:4050-9.

41. Zhang Y, Dube C, Gibert M Jr, Cruickshanks N, Wang B, Coughlan M, et al. The p53 pathway in glioblastoma. Cancers. 2018;10:297. 
42. Eldin P, Papon L, Oteiza A, Brocchi E, Lawson TG, Mechti N. TRIM22 E3 ubiquitin ligase activity is required to mediate antiviral activity against encephalomyocarditis virus. J Gen Virol. 2009;90:536-45.

43. Sivaramakrishnan G, Sun Y, Rajmohan R, Lin VC. B30.2/SPRY domain in tripartite motif-containing 22 is essential for the formation of distinct nuclear bodies. FEBS Lett. 2009;583: 2093-9.

44. Rothwarf DM, Zandi E, Natoli G, Karin M. IKK-gamma is an essential regulatory subunit of the IkappaB kinase complex. Nature. 1998;395:297-300.
45. Hatakeyama S, Kitagawa M, Nakayama K, Shirane M, Matsumoto $\mathrm{M}$, Hattori $\mathrm{K}$, et al. Ubiquitin-dependent degradation of IkappaBalpha is mediated by a ubiquitin ligase $\mathrm{Skp} 1 / \mathrm{Cul} 1 / \mathrm{F}-$ box protein FWD1. Proc Natl Acad Sci USA. 1999;96:3859-63.

46. Niu J, Shi Y, Iwai K, Wu ZH. LUBAC regulates NF-kappaB activation upon genotoxic stress by promoting linear ubiquitination of NEMO. EMBO J. 2011;30:3741-53.

47. Liu J, Han C, Xie B, Wu Y, Liu S, Chen K, et al. Rhbdd3 controls autoimmunity by suppressing the production of IL- 6 by dendritic cells via K27-linked ubiquitination of the regulator NEMO. Nat Immunol. 2014;15:612-22. 